International Journal of Pure and Applied Mathematics

Volume 93 No. $3 \quad 2014,449-461$

ISSN: 1311-8080 (printed version); ISSN: 1314-3395 (on-line version)

url: http://www.ijpam.eu

doi: http://dx.doi.org/10.12732/ijpam.v93i3.13

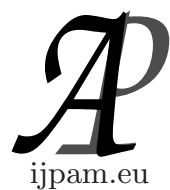

\title{
ON AN INHOMOGENEOUS BOUNDARY VALUE PROBLEM \\ WITH SHIFT INTO THE REGION FOR \\ THE CAUCHY-RIEMANN EQUATIONS WITH SPECTRAL PARAMETER
}

\author{
A.B. Amanzholova ${ }^{1}$, N.S. Imanbaev ${ }^{2}$, A.D. Niyazymbetov ${ }^{3}$ \\ ${ }^{1,2}$ Department of Mathematics \\ Akhmet Yasawi International Kazakh-Turkish University \\ 29, Sattarkhanov Street, 161200 Turkistan, KAZAKHSTAN \\ ${ }^{2}$ South Kazakhstan State Pedagogical Institute \\ Shymkent, KAZAKHSTAN
}

\begin{abstract}
In this paper we consider the spectral problem for the CauchyRiemann operator with Bicadze - Samarskii type boundary value conditions, reduced to a singular integral equation with continuous kernel. Moreover, we characterize those spectral parameters at which the inhomogeneous boundary value problem with shift into the region for Cauchy-Riemann equations is everywhere solvable in the class of continuous functions on the unit circle.
\end{abstract}

AMS Subject Classification: 32W50, 65N35

Key Words: Cauchy-Riemann operator, space of continuous functions, Fredholm, resolvent set, resolvent, problem with shift into the region, kernel

\section{Introduction}

Boundary value problems, arising in thermal conductivity, were formulated by V.A. Steklov (in 1922), and in gas dynamics - by F.I. Franklem (in 1956). In

Received: March 3, 2014

(c) 2014 Academic Publications, Ltd.

$\S$ Correspondence author url: www.acadpubl.eu 
1969 A.M. Nahushev introduced and studied several of problems of this type, and for their names proposed the term "with shift". In the same 1969 the paper of A.V. Bicadze and A.A. Samarskii was appeared, where the problem "with shift into the region" was studied [1]. Contents of recent publications have led to the realization of high quality novelty of boundary value problems with shifts for the theory of partial differential equations. In recent publications [2] for Bicadze - Lykov equations the problem with shift with Kober - Erdeyi and M. Saigo operators in the boundary condition was introduced, and questions about uniqueness and non uniqueness of solution of the problem at various functions and values of the constants, appearing in the boundary conditions, were studied. Abundance of publications, where the increasingly common situations are studied, sometimes produces the impression that the theory of boundary value problems "with shift" has been already completed. However, there are some less studied but important questions, in particular, the problem about eigenvalues or their analytical description, for example, using asymptotic expansions. Methods of function analysis and the method of reduction to the model equations by integral transformations, used recently, are insufficient to obtain such detailed information. On the other hand, one can hardly expect to get, for example, the solution of the eigenvalue problem for partial differential equations in as explicitly as it was done in a similar situation for ordinary differential equations. Generality of the above questions for partial differential equations forces further to impose a number of very severe restrictions on studied operators. Figuring out the correct formulation of the problem and studying the specific properties of the solutions for the "non-classical" equations it is convenient to begin by considering the idealized models, for example, by considering equations with constant coefficients. In the function space $C(|z| \leq 1)$ we consider operators, generated by the following Cauchy-Riemann differential operation:

$$
K \omega(z)=\frac{\partial \omega(z)}{\partial \bar{z}},
$$

where $z=x+i y, \bar{z}=x-i y, \frac{\partial}{\partial \bar{z}}=\frac{1}{2}\left(\frac{\partial}{\partial x}+i \frac{\partial}{\partial y}\right)$ in the set

$$
D(K) \subset\left\{\omega(z) \in C(|z| \leq 1), \frac{\partial \omega}{\partial \bar{z}} \in C(|z|<1)\right\} .
$$

We suppose that operator has a non empty resolvent set (). Without loss of generality, we assume that

$$
0 \in \rho(K),
$$

i.e. there exists a bounded operator -1 . In [3] the set of operators $K$ with the property (b) is fully described. 
Theorem (M. Otelbayev and .N. Shynybekov) [3]. For every linear operator with the condition (b) there exists a bounded operator G, mapping continuous functions on the circle $|z| \leq 1$ into holomorphic functions, that imaginary parts at $\mathrm{z}=0$ are equal to zero, and bounded functional $S(f)$ in the set of continuous functions on the circle $|z| \leq 1$, that uniquely defines the domain of $\mathrm{K}$ by the formula:

$$
\begin{gathered}
D(K)=\left\{\omega(z) \in C(|z| \leq 1), \frac{\partial \omega}{\partial \bar{z}} \in C(|z|<1)\right\}, \\
\operatorname{Re} \omega(z)=\operatorname{Re} G\left(\frac{\partial \omega}{\partial \bar{z}}\right),|z|=1 \\
\operatorname{Im} \omega(z)=\operatorname{Im} S\left(\frac{\partial \omega}{\partial \bar{z}}\right), z=0 .
\end{gathered}
$$

Conversely, a pair $\mathrm{G}$ and $\mathrm{S}$ are defined, for which (b) is true.

\section{Statement of the Problem}

Find complex values $\lambda \in$ - set of complex numbers, in which the operator equation

$$
K \omega(z)=\lambda \omega(z),|z|<1
$$

has a nonzero solution.

Aim of the paper. We consider the following spectral problem:

$$
\begin{gathered}
K \omega(z)=\frac{\partial \omega(z)}{\partial \bar{z}}=\lambda \omega(z),|z|<1, \\
\operatorname{Re} \omega(z)=\operatorname{Re}\left(\frac{1}{2 \pi i} \oint_{|\xi|=1} \frac{d \xi}{\xi-z} \cdot \oint_{|\tau|=r<1} \frac{\lambda \cdot \omega(\tau)}{\tau-\xi} d \tau|,| z \mid=1,\right. \\
\operatorname{Im} \omega(0)=\operatorname{Im}\left(\frac{1}{2 \pi i} \oint_{|\xi|=1} \frac{d \xi}{\xi} \cdot \oint_{|\tau|=r<1} \frac{\lambda \cdot \omega(\tau)}{\tau-\xi} d \tau\right),
\end{gathered}
$$

where $\lambda$ - spectral parameter.

The aim of the paper is to study the spectrum of elliptic operators, in particular, problems (1) - (3). 
Justification of result. The most profound results in this problem were found in [4]. In general, the spectrum of an elliptic operator is essentially determined by the spectral properties of the boundary operator G from M. Otelbaev and A.N. Shynybekov's theorem [3]. However, ascertainment dependence of spectrum of the operator $\mathrm{K}$ in the original terms of the boundary conditions is relevant (unsolved) problem. From the general results such facts are not tracked, so we have to attract deeper methods associated with the specifics of the specific boundary conditions. Description of the general regular boundary value problems for Cauchy-Riemann differential expression was developed by J. F. Neyman, M.I.Vishik, A.A. Dezin and M. Otelbaev. The problem (1) (3) is a problem with shift into the region, which is called Bicadze - Samarskii type, i.e. (regular) and similar problems for Cauchy-Riemann operation were described in 1982 by M. Otelbaev and A.N. Shynybekov [3]. In [5]-[6] question of stability of basis property of systems of eigenvectors and associated vectors of the Schr?dinger operator with regular but not strongly regular boundary value conditions has been studied. In [7] the spectral questions of problem with shift for ordinary differential operator of the third order on the interval in the space of square integrable functions have been investigated. From another point of view, the question of solvability and behavior of the solution of the boundary value problem for a generalized Cauchy-Riemann equation were deeply studied in $[8],[9],[10]$. Boundary value problems for the generalized Cauchy-Riemann system with non smooth coefficients were investigated in [11]-[12].

\section{Main Result}

Theorem 1. A solution of the eigenvalue problem of Cauchy - Riemann differential operator (1) - (3) is defined by the following formula:

$$
\omega(z)=\frac{e^{\lambda \bar{z}}}{2 \pi i} \oint_{|\xi|=1} u(\xi) \frac{\xi+z}{\xi-z} \cdot \frac{d \xi}{\xi}+i C e^{\lambda \bar{z}}+u(z) e^{\lambda \bar{z}},
$$

moreover, for the real function $u(z)$ on the circle $|z|=1$ the following singular integral equation holds:

$$
\begin{gathered}
a(z) u(z)-\frac{b(z)}{\pi i} \oint_{|\xi|=1} \frac{u(\xi)}{\xi-z} d \xi+\oint_{|\xi|=1} H(z, \xi) u(\xi) d \xi+ \\
+\frac{1}{2 \pi i} \oint_{|\xi|=1} \frac{d \xi}{\xi}\left(i\left(e^{\lambda \bar{z}}-e^{\bar{\lambda} z}\right)-\pi\left(\bar{\lambda}+\lambda+\bar{\lambda} e^{\bar{\lambda} r^{2} z}-\lambda e^{\lambda r^{2} \bar{z}}\right)\right) \times
\end{gathered}
$$




$$
\begin{aligned}
& \times\left[\oint_{\lfloor\tau \mid=r<1} u(\tau)\left(\frac{\lambda e^{\lambda \bar{\tau}}}{\tau-\xi}+\frac{\xi r^{2}}{\tau} \cdot \frac{\bar{\lambda} e^{\bar{\lambda} \tau}}{\tau-r^{2} \xi}\right) \cdot d \tau \mid-\frac{1}{2 \pi i} \oint_{|\xi|=1} \frac{d \xi}{\xi-z} \times\right. \\
& \times\left\{\left[\oint_{\lfloor\tau \mid=r<1} u(\tau)\left(\frac{\lambda e^{\lambda \bar{\tau}}}{\tau-\xi}+\frac{\xi r^{2}}{\tau} \cdot \frac{\bar{\lambda} e^{\bar{\lambda} \tau}}{\tau-r^{2} \xi}\right) \cdot d \tau \mid\right\}=0,|z|=1,\right.
\end{aligned}
$$

where

$$
\begin{gathered}
a(z)=e^{\lambda \bar{z}}+e^{\bar{\lambda} z} \\
b(z)=\frac{(\lambda+\bar{\lambda}) \pi i-e^{\lambda \bar{z}}+e^{\bar{\lambda} z}}{2}, \\
H(z, \xi)=\left(i\left(e^{\lambda \bar{z}}-e^{\bar{\lambda} z}\right)-\pi\left(\lambda e^{\lambda r^{2} \bar{z}}-\bar{\lambda} e^{\bar{\lambda} r^{2} z}-\bar{\lambda}-\lambda\right) \cdot \frac{1}{\xi}((\lambda+\bar{\lambda})-\right. \\
\left.-\lambda e^{\lambda r^{2} \bar{\xi}}-\bar{\lambda} e^{\bar{\lambda} r^{2} \xi}\right)-\frac{1}{\xi-z} \\
\frac{z}{\xi}\left(\bar{\lambda} e^{\bar{\lambda} r^{2} \xi}+\frac{\bar{\lambda}-\lambda}{2}+\bar{\lambda}-\frac{e^{\lambda \bar{z}}+e^{\bar{\lambda} z}}{2 \pi i}\right)+ \\
+\frac{\lambda e^{\lambda r^{2} \bar{\xi}}}{\xi-z}-\frac{\lambda e^{\lambda r^{2} \bar{z}}+\bar{\lambda} e^{\bar{\lambda} r^{2} \xi}}{2} \cdot \frac{\xi+z}{\xi-z} \cdot \frac{1}{\xi}
\end{gathered}
$$

$H(z, \xi)$ - continuous kernel. The constant from the general solution can be introduced as follows:

$$
C=\operatorname{Im}\left\{\lambda \oint_{|\xi|=1} u(\xi)\left(1-e^{\lambda r^{2} / \xi} \frac{d \xi}{\xi}+\frac{\lambda}{2 \pi i} \oint_{|\xi|=1} \frac{d \xi}{\xi} \cdot \oint_{|\tau|=r<1} \frac{e^{\lambda \bar{\tau}}}{\tau-\xi} u(\tau) d \tau\right\} .\right.
$$

Proof. The general solution of partial differential equation has the form:

$$
\omega(z)=\Phi(z) e^{\lambda \bar{z}},
$$

where $\Phi(z)$ - holomorphic function in the circle $|z|<1$. Indeed, multiplying both sides of (1) by $e^{-\lambda \bar{z}}$, we get

$$
e^{-\lambda \bar{z}} \frac{\partial \omega(z)}{\partial \bar{z}}-\lambda e^{-\lambda \bar{z}} \omega(z)=0 .
$$

Since

$$
\frac{\partial e^{-\lambda \bar{z}}}{\partial \bar{z}}=-\lambda e^{-\lambda \bar{z}},
$$


we have the equality

$$
\frac{\partial}{\partial \bar{z}}\left(e^{-\lambda \bar{z}} \omega(z)\right)=0
$$

Consequently, product $e^{-\lambda \bar{z}} \omega(z)$ is a holomorphic function in the circle $|z|<$ 1. The holomorphic function $\Phi(z)$ is expressed by Schwartz integral formula through its real part as follows:

$$
\Phi(z)=\frac{1}{2 \pi i} \oint_{|\xi|=1} u(\xi) \frac{\xi+z}{\xi-z} \cdot \frac{d \xi}{\xi}+i C,|z|<1,
$$

where $u(\xi)=\operatorname{Re} \Phi(\xi),|\xi|=1, C=\operatorname{Im} \Phi(0)$. Then the formula (4) has the form:

$$
\omega(z)=\frac{e^{\lambda \bar{z}}}{2 \pi i} \oint_{|\xi|=1} u(\xi) \frac{\xi+z}{\xi-z} \cdot \frac{d \xi}{\xi}+i C e^{\lambda \bar{z}},|z|<1 .
$$

The last formula is a general solution of the equation (1), that we put into the boundary condition (2). Using Sokhotskii - Plemelj formula [13], we find boundary value of the solution when $|z|=1$ :

$$
\omega(z)=\frac{e^{\lambda \bar{z}}}{2 \pi i} \oint_{|\xi|=1} u(\xi) \frac{\xi+z}{\xi-z} \cdot \frac{d \xi}{\xi}+i C e^{\lambda \bar{z}}+u(z) e^{\lambda \bar{z}}
$$

Then we obtain

$$
\begin{gathered}
\operatorname{Re}\left\{\frac{e^{\lambda \bar{z}}}{2 \pi i} \oint_{|\xi|=1} u(\xi) \frac{\xi+z}{\xi-z} \cdot \frac{d \xi}{\xi}+i C e^{\lambda \bar{z}}+u(z) e^{\lambda \bar{z}}\right\}= \\
=\operatorname{Re}\left\{\frac{e^{\lambda \bar{z}}}{2 \pi i} \oint_{|\xi|=1} \frac{d \xi}{\xi-z} \cdot \oint_{|\tau|=r<1} \frac{\lambda d \tau}{\tau-\xi} \cdot \frac{e^{\lambda \bar{z}}}{2 \pi i} \oint_{|\theta|=1} u(\theta) \frac{\theta+\tau}{\theta-\tau} \cdot \frac{d \theta}{\theta}+\right. \\
+\frac{1}{2 \pi i} \oint_{|\xi|=1} \frac{d \xi}{\xi-z} \cdot \oint_{|\tau|=r<1} \frac{\lambda d \tau}{\tau-\xi} i \cdot C e^{\lambda \bar{z}}+ \\
+\frac{1}{2 \pi i} \oint_{|\xi|=1} \frac{d \xi}{\xi-z} \cdot \oint_{|\tau|=r<1} \frac{\lambda d \tau}{\tau-\xi} \cdot u(\tau) e^{\lambda \bar{z}}+
\end{gathered}
$$




$$
\begin{gathered}
+\frac{1}{2 \pi i} \oint_{|\xi|=1} \frac{d \xi}{\xi-z} \cdot \oint_{|\tau|=r<1} \frac{\lambda d \tau}{\tau-\xi} \cdot \frac{1}{2 \pi i} \oint_{|t|=1} \frac{d t}{t-\tau} \cdot \frac{e^{\lambda \bar{t}}}{2 \pi i} \oint_{|v|=1} u(\mathrm{v}) \frac{\mathrm{v}+\mathrm{t}}{\mathrm{v}-\mathrm{t}} \cdot \frac{d \mathrm{v}}{\mathrm{v}}+ \\
\frac{1}{2 \pi i} \oint_{|\xi|=1} \frac{d \xi}{\xi-z} \cdot \oint_{|\tau|=r<1} \frac{\lambda d \tau}{\tau-\xi} \cdot \frac{1}{2 \pi i} \oint_{|t|=1} \frac{d t}{t-\tau} i \cdot C e^{\lambda \bar{t}}+\frac{1}{2 \pi i} \oint_{|\xi|=1} \frac{d \xi}{\xi-z} \cdot \oint_{|\tau|=r<1} \frac{\lambda d \tau}{\tau-\xi} \\
\left.\times \frac{1}{2 \pi i} \oint_{|t|=1} e^{\lambda \bar{t}} u(t) \frac{d t}{t-\tau}\right\},|z|=1 .
\end{gathered}
$$

Due to the Poincare - Bertrand formula [13], changing order of integration, we rewrite the last equality as follows:

$$
\begin{aligned}
& \operatorname{Re}\left\{\frac{e^{\lambda \bar{z}}}{2 \pi i} \oint_{|\xi|=1} u(\xi) \frac{\xi+z}{\xi-z} \cdot \frac{d \xi}{\xi}+i C e^{\lambda \bar{z}}+u(z) e^{\lambda \bar{z}}\right\}= \\
& =\operatorname{Re}\left\{\frac{1}{2 \pi i} \oint_{|\theta|=1} u(\theta) \frac{d \theta}{\theta}+\oint_{|\xi|=1} \frac{\lambda d \xi}{\xi-z} \cdot \frac{1}{2 \pi i} \oint_{|\tau|=r<1} e^{\lambda \bar{\tau}} \frac{\theta+\tau}{\theta-\tau} \cdot \frac{d \tau}{\tau-\xi}+\right. \\
& +\frac{i \lambda C}{2 \pi i} \oint_{|\xi|=1} \frac{\lambda d \xi}{\xi-z} \oint_{|\tau|=r<1} e^{\lambda \bar{\tau}} \frac{d \tau}{\tau-\xi}+\frac{\lambda}{2 \pi i} \oint_{|\xi|=1} \frac{\lambda d \xi}{\xi-z} \cdot \oint_{|\tau|=r<1} e^{\lambda \bar{\tau}} u(\tau) \frac{d \tau}{\tau-\xi}+ \\
& +\frac{1}{2 \pi i} \oint_{|v|=1} u(\mathrm{v}) \frac{d \mathrm{v}}{\mathrm{v}} \cdot \oint_{|\xi|=1} \frac{\lambda d \xi}{\xi-z} \cdot \frac{1}{2 \pi i} \oint_{|\tau|=r<1} \frac{d \tau}{\tau-\xi} \cdot \frac{1}{2 \pi i} \oint_{|\mathrm{t}|=1} e^{\lambda \bar{t}} \frac{\mathrm{v}+\mathrm{t}}{\mathrm{v}-\mathrm{t}} \cdot \frac{d t}{t-\tau}+ \\
& +\frac{i \lambda C}{2 \pi i} \oint_{|\xi|=1} \frac{\lambda d \xi}{\xi-z} \oint_{|\tau|=r<1} \frac{d \tau}{\tau-\xi} \cdot \frac{1}{2 \pi i} \oint_{|\mathrm{t}|=1} e^{\lambda \bar{t}} \frac{d \mathrm{t}}{\mathrm{t}-\tau}+\frac{\lambda}{2 \pi i} \oint_{|\mathrm{t}|=1} e^{\lambda \bar{t}} u(t) d t \times \\
& \left.\times \oint_{|\xi|=1} \frac{\lambda d \xi}{\xi-z} \cdot \frac{1}{2 \pi i} \oint_{|\tau|=r<1} \frac{d \tau}{\tau-\xi} \cdot \frac{1}{2 \pi i} \oint_{|\tau|=r<1} \frac{d \tau}{(\tau-\xi)(t-\tau)}\right\},|z|=1 .
\end{aligned}
$$

Since $\tau \cdot \bar{\tau}=r^{2}, \bar{\tau}=\frac{r^{2}}{\tau}, \bar{t}=\frac{1}{t}, \bar{v}=\frac{1}{v}, \bar{z}=\frac{1}{z}, \bar{\theta}=\frac{1}{\theta}, \bar{\xi}=\frac{1}{\xi}$, then using residues, one can calculate integrals in (5), not containing an unknown function $u(\xi)$. The following lemma holds: Lemma. Residue at the singular point $\tau=0$ from the integral:

$$
\frac{1}{2 \pi i} \oint_{|\tau|=r<1} e^{\lambda \bar{\tau}} \frac{d \tau}{\tau-A}
$$


can be calculated by the formula:

$$
\underset{\tau=0}{\operatorname{res}} \frac{e^{\lambda \bar{\tau}}}{\tau-A}=\frac{-\lambda r^{2}}{A}-\frac{-\lambda^{2} r^{4}}{2 ! A^{2}}-\frac{-\lambda^{3} r^{6}}{3 ! A^{3}}-\ldots=1-e^{\frac{\lambda r^{2}}{A}} .
$$

According to this lemma, we calculate residues of functions into the integral, which have singularity at the point $\tau=0$. Residues at other singular points of the function into the integral are easy calculated, since singularities have the form of the first order poles [14]:

$$
\begin{aligned}
& I_{1}=\frac{1}{2 \pi i} \oint_{|\theta|=1} u(\theta) \frac{d \theta}{\theta}+\oint_{|\xi|=1} \frac{d \xi}{\xi-z} \cdot \oint_{|\tau|=r<1} e^{\lambda \bar{\tau}} \frac{\theta+\tau}{\theta-\tau} \cdot \frac{d \tau}{\tau-\xi}= \\
& =-\pi i \oint_{|\theta|=1} u(\theta) \frac{d \theta}{\theta}\left[\frac{2 \theta}{\theta-z} \cdot\left(e^{\frac{\lambda r^{2}}{\theta}}-1\right)-\frac{\theta+z}{\theta-z}\left(e^{\frac{\lambda r^{2}}{z}}-1\right)\right], \\
& I_{2}=i C \oint_{|\xi|=1} \frac{d \xi}{\xi-z} \cdot \oint_{|\tau|=r<1} e^{\lambda \bar{\tau}} \frac{\theta+\tau}{\theta-\tau} \cdot \frac{d \tau}{\tau-\xi}=2 \pi^{2} i C\left(e^{\frac{\lambda r^{2}}{\theta}}-1\right) \text {, } \\
& I_{3}=\frac{1}{2 \pi i} \oint_{|\mathrm{v}|=1} u(\mathrm{v}) \frac{d \mathrm{v}}{\mathrm{v}} \oint_{|\xi|=1} \frac{d \xi}{\xi-z} \cdot \oint_{|\tau|=r<1} \frac{d \tau}{\tau-\xi} \cdot \frac{1}{2 \pi i} \oint_{|\mathrm{t}|=1} e^{\lambda \bar{t}} \frac{\mathrm{v}+\mathrm{t}}{\mathrm{v}-\mathrm{t}} \frac{d t}{t-\tau}= \\
& =\frac{1}{2 \pi i} \oint_{|\mathrm{v}|=1} u(\mathrm{v}) \frac{d \mathrm{v}}{\mathrm{v}} \oint_{|\xi|=1} \frac{d \xi}{\xi-z} \cdot \oint_{|\tau|=r<1} \frac{d \tau}{\tau-\xi} \cdot\left\{\frac{2 \mathrm{v}}{\mathrm{v}-\mathrm{t}} \cdot\left(e^{\frac{\lambda}{\mathrm{v}}}-1\right)-\right. \\
& \left.-\frac{\mathrm{v}+\tau}{\mathrm{v}-\tau} \cdot\left(e^{\frac{\lambda}{\tau}}-1\right)+\frac{\mathrm{v}+\tau}{\mathrm{v}-\tau} \cdot e^{\frac{\lambda}{\tau}}-\frac{2 \mathrm{v}}{\mathrm{v}-\tau} \cdot e^{\frac{\lambda}{\mathrm{v}}}\right\}=0 \\
& I_{4}=\lambda i C \frac{1}{2 \pi i} \oint_{|\xi|=1} \frac{d \xi}{\xi-z} \cdot \oint_{|\tau|=r<1} \frac{d \tau}{\tau-\xi} \cdot \oint_{|t|=1} e^{\lambda \bar{t}} \frac{d t}{t-\tau}= \\
& =i C \frac{\lambda}{2 \pi i} \oint_{|\xi|=1} \frac{d \xi}{\xi-z} \cdot \oint_{|\tau|=r<1} \frac{d \tau}{\tau-\xi} \cdot 2 \pi i\left[\left(1-e^{\frac{\lambda}{\tau}}\right)+e^{\frac{\lambda}{\tau}}\right]=0, \\
& I_{5}=\frac{\lambda}{2 \pi i} \oint_{|\mathrm{t}|=1} e^{\lambda \bar{t}} u(t) d t \oint_{|\xi|=1} \frac{\lambda d \xi}{\xi-z} \cdot \frac{1}{2 \pi i} \oint_{|\tau|=r<1} \frac{d \tau}{(\tau-\xi)(t-\tau)}=0 .
\end{aligned}
$$


So, equality (5) can be rewritten as:

$$
\begin{gathered}
\operatorname{Re}\left\{\frac{e^{\lambda \bar{z}}}{2 \pi i} \oint_{|\xi|=1} u(\xi) \frac{\xi+z}{\xi-z} \cdot \frac{d \xi}{\xi}+i C e^{\lambda \bar{z}}+u(z) e^{\lambda \bar{z}}\right\}= \\
=\operatorname{Re} \frac{\lambda}{2 \pi i}\left\{-\pi i \oint_{|\theta|=1} u(\theta) \frac{d \theta}{\theta} \cdot\left[\frac{2 \theta}{\theta-z} \cdot\left(e^{\frac{\lambda r^{2}}{\theta}}-1\right)-\frac{\theta+z}{\theta-z}\left(e^{\frac{\lambda r^{2}}{z}}-1\right)\right]+\right. \\
\left.+2 \pi^{2} i C\left(1-e^{\frac{\lambda r^{2}}{z}}\right)+\oint_{|\xi|=1} \frac{\lambda d \xi}{\xi-z} \oint_{|\tau|=r<1} \frac{e^{\lambda \tau}}{\tau-\xi} u(\tau) d \tau\right\},|z|=1 .
\end{gathered}
$$

We write the real part of a complex number in the form of half-sum of a complex number and its conjugate, then we obtain the relation when $|z|=1$ :

$$
\begin{gathered}
\frac{e^{\lambda \bar{z}}}{2 \pi i} \oint_{|\xi|=1} u(\xi) \frac{\xi+z}{\xi-z} \cdot \frac{d \xi}{\xi}-\frac{e^{\bar{\lambda} z}}{2 \pi i} \oint_{|\xi|=1} u(\xi) \frac{\xi+z}{\xi-z} \cdot \frac{d \xi}{\xi}+ \\
+i C e^{\lambda \bar{z}}-i C e^{\bar{\lambda} z}+u(z) e^{\lambda \bar{z}}+u(z) e^{\bar{\lambda} z}= \\
=\frac{-\lambda}{2} \oint_{|\xi|=1} u(\xi) \frac{d \xi}{\xi-z} \cdot 2 e^{\frac{\lambda r}{\xi}}+\bar{\lambda} \oint_{|\xi|=1} e^{\bar{\lambda} r^{2} \xi} u(\xi) \frac{1}{\frac{z-\xi}{\xi \cdot z}} \cdot(-1) \frac{d \xi}{\xi^{2}}+ \\
+\lambda \oint_{|\xi|=1} u(\xi) \frac{d \xi}{\xi-z}+\bar{\lambda} \oint_{|\xi|=1} u(\xi) \frac{1}{\frac{z-\xi}{\xi}} \cdot(-1) \frac{d \xi}{\xi^{2}}+ \\
+\frac{\lambda}{2} \oint_{|\xi|=1} u(\xi) \frac{\xi+z}{\xi-z} \cdot \frac{d \xi}{\xi} \cdot e^{\lambda r^{2} \bar{z}}+\frac{\bar{\lambda}}{2} \oint_{|\xi|=1} u(\xi) \frac{\xi+z}{\xi-z} \cdot \frac{d \xi}{\xi} \cdot e^{\bar{\lambda} r^{2} z}- \\
-\frac{\lambda}{2} \oint_{|\xi|=1} u(\xi) \frac{\xi+z}{\xi-z} \cdot \frac{d \xi}{\xi}+\frac{\bar{\lambda}}{2}-\pi \lambda C e^{\lambda r^{2} \bar{z}}+\pi \bar{\lambda} C e^{\bar{\lambda} r^{2} z}+\pi \lambda C+\pi \bar{\lambda} C+ \\
+\frac{\lambda}{2 \pi i} \oint_{|\xi|=1} \frac{\lambda d \xi}{\xi-z} \oint_{|\tau|=r<1} \frac{e^{\lambda \bar{\tau}}}{\tau-\xi} u(\tau) d \tau \\
+\frac{\bar{\lambda}}{2 \pi i} \oint_{|\xi|=1} \frac{1}{\frac{\xi-z}{\xi z}} \cdot(-1) \frac{d \xi}{\xi^{2}} \oint_{|\tau|=r<1} \frac{e^{\bar{\lambda} \tau}}{\frac{r^{2}}{\tau}-\frac{1}{\xi}} u(\tau) \frac{-r^{2}}{\tau^{2}} d \tau .
\end{gathered}
$$


The last relation is transformed to the form:

$$
\begin{gathered}
\frac{e^{\lambda \bar{z}}-e^{\bar{\lambda} z}}{2 \pi i} \oint_{|\xi|=1} u(\xi) \frac{\xi+z}{\xi-z} \cdot \frac{d \xi}{\xi}+i C\left(e^{\lambda \bar{z}}-e^{\bar{\lambda} z}\right)+\left(e^{\lambda \bar{z}}+e^{\bar{\lambda} z}\right) u(z)= \\
=-\lambda \oint_{|\xi|=1} \frac{e^{\lambda r^{2} \bar{\xi}}}{\xi-z} u(\xi) d \xi+\bar{\lambda} \oint_{|\xi|=1} \frac{e^{\bar{\lambda} r^{2} \xi}}{\xi-z} \cdot \frac{z}{\xi} u(\xi) d \xi+\lambda \oint_{|\xi|=1} \frac{1}{\xi-z} u(\xi) d \xi+ \\
+\bar{\lambda} \oint_{|\xi|=1} \frac{1}{\xi-z} \cdot \frac{z}{\xi} u(\xi) d \xi+\left(\frac{\lambda}{2} e^{\lambda r^{2} \bar{z}}+\frac{\bar{\lambda}}{2} e^{\bar{\lambda} r^{2} z}-\frac{\lambda}{2}+\frac{\bar{\lambda}}{2}\right) \oint_{|\xi|=1} u(\xi) \frac{\xi+z}{\xi-z} \cdot \frac{d \xi}{\xi}+ \\
+\pi C\left(-\lambda e^{\lambda r^{2} \bar{z}}+\bar{\lambda} e^{\bar{\lambda} r^{2} z}+\lambda+\bar{\lambda}\right)+\frac{\lambda}{2 \pi i} \oint_{|\xi|=1} \cdot \frac{d \xi}{\xi-z} \oint_{|\tau|=r<1} \frac{e^{\lambda \bar{\tau}}}{\tau-\xi} u(\tau) d \tau- \\
-\frac{\bar{\lambda}}{2 \pi i} \oint_{|\xi|=1} \cdot \frac{1}{\xi-z} \cdot \frac{\mathrm{z}}{\xi} d \xi \oint_{|\tau|=r<1} \frac{e^{\bar{\lambda} \tau}}{\tau-r^{2} \xi} \cdot \frac{\xi r^{2}}{\tau} u(\tau) d \tau,|z|=1 .
\end{gathered}
$$

Highlighting the singular part, we can write the resulting relation in a canonical form:

$$
\begin{gathered}
\left(e^{\lambda \bar{z}}+e^{\bar{\lambda} z}\right) u(z)+\frac{e^{\lambda \bar{z}}-e^{\bar{\lambda} z}-(\lambda+\bar{\lambda}) \pi i}{2} \cdot \frac{1}{\pi \mathrm{i}} \oint_{|\xi|=1} \frac{u(\xi)}{\xi-z} d \xi+ \\
+\oint_{|\xi|=1} u(\xi)\left\{\frac{e^{\lambda \bar{z}}-e^{\bar{\lambda} z}}{2 \pi i} \cdot \frac{1}{\xi-z} \cdot \frac{z}{\xi}+\frac{\lambda e^{\lambda r^{2} \bar{\xi}}}{\xi-z}-\frac{\bar{\lambda} e^{\bar{\lambda} r^{2} \xi}}{\xi-z} \cdot \frac{z}{\xi}-\frac{\bar{\lambda}}{\xi-z} \cdot \frac{z}{\xi}-\frac{\bar{\lambda}-\lambda}{2} \frac{1}{\xi-z} \cdot \frac{z}{\xi}-\right. \\
\left.-\frac{\lambda e^{\lambda r^{2} \bar{z}}+\bar{\lambda} e^{\bar{\lambda} r^{2} z}}{2} \frac{1}{\xi-z} \cdot \frac{z}{\xi}\right\} d \xi+C\left(i e^{\lambda \bar{z}}-i e^{\bar{\lambda} z}+\pi \lambda e^{\lambda r^{2} \bar{z}}-\pi \bar{\lambda} e^{\bar{\lambda} r^{2} z}-\pi \lambda-\pi \bar{\lambda}\right)- \\
-\frac{1}{2 \pi i} \oint_{|\xi|=1} \cdot \frac{d \xi}{\xi-z}\left\{\oint_{|\tau|=r<1} \frac{\lambda e^{\lambda \bar{\tau}}}{\tau-\xi} \cdot u(\tau) d \tau-\frac{z}{\xi} \oint_{|\tau|=r<1} \frac{\bar{\lambda} e^{\bar{\lambda} \tau}}{\tau-r^{2} \xi} \cdot \frac{\xi r^{2}}{\tau} \cdot u(\tau) d \tau\right\} \\
=0, \quad|z|=1 . \quad(7)
\end{gathered}
$$

In order to eliminate $\mathrm{C}$ from (7) we use the boundary value condition (3). We calculate integrals, appearing in the obtained equality after using the boundary value condition (3), and not containing an unknown function $u(\xi)$. Analogously, 
substituting the obtained values of integrals, and grouping similar terms, we get the formula for the real constants:

$$
\begin{gathered}
\left(e^{\lambda \bar{z}}+e^{\bar{\lambda} z}\right) u(z)-\frac{(\lambda+\bar{\lambda}) \pi i-e^{\lambda \bar{z}}-e^{\bar{\lambda} z}}{2} \cdot \frac{1}{\pi \mathrm{i}} \oint_{|\xi|=1} \frac{u(\xi)}{\xi-z} d \xi+ \\
+\oint_{|\xi|=1} u(\xi)\left\{\frac{1}{\xi}(\lambda+\bar{\lambda})-\lambda e^{\lambda r^{2} \bar{\xi}}-\bar{\lambda} e^{\bar{\lambda} r^{2} \xi} \cdot\left(i\left(e^{\lambda \bar{z}}-e^{\bar{\lambda} z}\right)+\pi\left(\lambda e^{\lambda r^{2} \bar{z}}-\bar{\lambda} e^{\bar{\lambda} r^{2} z}-\lambda+\bar{\lambda}\right)\right)+\right. \\
\left.+\frac{\lambda e^{\frac{\lambda r^{2}}{\xi}}}{\xi-z}-\frac{1}{\xi-z} \cdot \frac{z}{\xi} \cdot\left(\bar{\lambda} e^{\bar{\lambda} r^{2} \xi}+\bar{\lambda}+\frac{\bar{\lambda}-\lambda}{2}-\frac{e^{\lambda \bar{z}}-e^{\bar{\lambda} z}}{2 \pi i}\right)-\frac{\lambda e^{\lambda r^{2} \bar{z}}-\bar{\lambda} e^{\bar{\lambda} r^{2} z}}{2} \frac{\xi+z}{\xi-z} \cdot \frac{1}{\xi}\right\} d \xi+ \\
+\frac{1}{2 \pi i} \oint_{|\xi|=1} \frac{d \xi}{\xi}\left[\oint_{\lfloor\tau \mid=r<1} u(\tau)\left(\frac{\lambda e^{\lambda \bar{\tau}}}{\tau-\xi}+\frac{\xi r^{2}}{\tau} \cdot \frac{\lambda e^{\bar{\lambda} \tau}}{\tau-r^{2} \xi}\right) d t \mid\left(i\left(e^{\lambda \bar{z}}-e^{\bar{\lambda} z}\right)\right)-\right. \\
-\pi\left(\bar{\lambda}+\lambda+\bar{\lambda} e^{\bar{\lambda} r^{2} z}-\lambda e^{\lambda r^{2} \bar{z}}\right)-\frac{1}{2 \pi \mathrm{i}} \oint_{|\xi|=1} \frac{d \xi}{\xi-z} \cdot\left\{\oint _ { | \tau | = r < 1 } u ( \tau ) \left(\frac{\lambda e^{\lambda \bar{\tau}}}{\tau-\xi}-\right.\right. \\
\left.\left.-\frac{z}{\xi} \cdot \frac{\xi r^{2}}{\tau} \cdot \frac{\lambda e^{\bar{\lambda} \tau}}{\tau-r^{2} \xi}\right) d \tau\right\}=0, \quad|z|=1 .
\end{gathered}
$$

Thus, the problem (1) - (3) is reduced to a singular integral equation (8). Theorem 1 is proved. In [15],[16],[17] the equivalence of similar boundary value problems with shift to a singular integral equation has been proved.

\section{References}

[1] Nahushev .. Problems with shift for Partial Equations. - M.: Nauka, -287p. (2006)

[2] E.Yi.Arlanova On the basis of eigenfunctions of ordinary differential operators with integral boundary conditions Vestnik Samarskogo State Techn. Universita. Ser. Phys.-Math. Sciences. 4(29).- P.26-36. (2012)

[3] M.O.Otelbayev and A.N.Shynybekov On exact Bicadze - Samarskii type problems. Doklady AN SSSR V.265, 4 P.815-819. (1982)

[4] V.A.Mihailets Spectral Problems with General Boundary Value Conditions: Autoref.doct.phys.-math.nauk.-Kiev, - 29p. (1989) 
[5] Sadybekov .., Imanbaev N.S. On the Basis Property of Root Functions of a Periodic Problem with an Integral Perturbation of the Boundary Condition Differential Equations V.48, 6. (2012). P.896-900.

[6] Imanbaev N.S., Kalimbetov B.T. and Sarsenbi A.M. Study basicity of root functions of the Schrodinger operator with a non-local perturbation. International Journal of Pure and Applied Mathematics. V.89, 3, P.433-438. (2013)

[7] Imanbaev N.S., Kanguzhin B.E. and Kalimbetov B.T. On zeros of the characteristic determinant of the spectral problem for a third order differential operator on a segment with nonlocal boundary conditions. Advances in Difference Equations. MS ID: 4768465999052350, (2013)

[8] Ying Wang and Yufeng Wang, Two Boundary-Value Problems for the Cauchy-Riemann Equation in a Sector. Complex Analysis and Operator Theory. V.6, 6, P.1121-1138. (2012)

[9] Timopheyev A.Yi. Boundary value problem for the generalized CauchyRiemann equations in spaces described by the modulus of continuity. Uphymskii Mathem. Journal. V.4, 1 P.146-152. (2012)

[10] Ilchukov .S. On behavior of solutions of the boundary value problem for a gen-eralized Cauchy-Riemann equation. Vestnik Udmurtskogo University. Ser. Mathem. Mech. Computer Science. V. 2. (2013)

[11] Ospanov .N. and Otelbayev M. Boundary value problems for generalized Cauchy - Riemann system with non smooth coefficients. Doklady N SSSR. V.283. 1. P.46-49. (1985)

[12] Ospanov .N. and Otelbayev M. On the generalized Cauchy-Riemann system with non-smooth coefficients. Izvestya VUZov. Mathem., 3. P.48-56. (1989)

[13] Mushelishvili N.I. Singular Integral Equations. - .,-511p. (1968)

[14] Shabat B.V. Introduction to Complex Analysis. - ., -320p. (1976)

[15] Mihlin S.G. Singular integral equations. Uspehi mathem. nauk. 3 (25). P.30-111. (1948)

[16] Imanbayev N.S. On noethericity a singular integral equation. Izvestya NAN RK. Physical and Mathematical series. Almaty. 1 (221). P.90-92. (2002) 
[17] Imanbayev N.S. On the eigenvalue problem of Cauchy-Riemann operator with Bitsadze - Samarskii type boundary value conditions. Vestnik Abay KazNPU. Series "Physical and Mathematical Sciences", Almaty, 3 (43). P.86-93. (2013). 
